Editors: Lawrence Rosenblum and Michael Macedonia

\title{
Enabling a Continuum of Virtual Environment Experiences
}

Larry Davis, Jannick Rolland, Felix HamzaLup, and Yonggang Ha

University of Central Florida

Jack Norfleet

US Army Simulation, Training, and Instrumentation Command

Celina Imielinska

Columbia University
W e define a virtual environment as a set of surroundings that appear to a user through computer-generated sensory stimuli. The level of immersion - or sense of being in another world - that a user experiences within a VE relates to how much stimuli the computer delivers to the user. Thus, you can classify VEs along a virtuality continuum, illustrated in Figure 1, which ranges from the real world to an entirely computer-generated environment. In this article, we present a technology that allows seamless transitions between levels of immersion in VEs.

Milgram and Kishino first proposed the concept of a virtuality continuum in the context of visual displays. ${ }^{1}$ The concept of a virtuality continuum extends to multimodal VEs, which combine multiple sensory stimuli, including 3D sound and haptic capability, leading to a multidimensional virtuality continuum. Emerging applications will benefit from multiple levels of immersion, requiring innovative multimodal technologies and the ability to traverse the multidimensional virtuality continuum. (See the "MagicBook" sidebar for an example application.)

\section{Alice's adventures in virtuality}

The experience of entering VEs resembles Lewis Carroll's Alice's Adventures in Wonderland. We present a metaphor-based on this classic children's tale-for transitioning between virtual realities. In the book, Alice seeks an escape from reality. The escape begins with her seeing the talking white rabbit. The rabbit is part of her reality, yet clearly doesn't belong. This marks her departure from reality. However, because she experiences the rabbit as part of her real world, this is Alice's augmented reality. Next, Alice becomes increasingly a part of and immersed in Wonderland. At this stage, Alice is becoming more familiar with this fantastic environment. However, she still feels as though she is an outsider within the world.
Her feeling of being an outsider relates to our notion of augmented virtuality, where real stimuli are inserted as part of a larger virtual world. That is, Alice is both real and part of a virtual world. Finally, after attempting to relate the fantasy world with the real world by asking directions to the Cheshire Cat, Alice attends the Mad Hatter's Tea Party, plays croquet with the Queen of Hearts, and has a provoking discussion with the Mock Turtle and the Gryphon. At this point, Alice has totally accepted the utter ridiculousness of Wonderland and embraces the craziness. She exists totally within her virtual reality.

\section{Traversing the continuum}

Alice's experience and dream of Wonderland were compelling to her. The environment was interactive, entertaining, and in some places educational. Furthermore, Alice traversed the levels of the virtuality continuum directly. Her experiences inspired us to develop technology that enables transitions through virtuality as seamlessly and naturally as possible.

Technology based on head-mounted projection displays (HMPDs) has recently matured as complex integrated systems. ${ }^{2}$ Here, we demonstrate how the Artificial Reality Center (ARC) uses HMPDs to help users span the virtuality continuum. In the ARC, users' navigation between the levels of immersion occurs on the basis of where users position themselves within the real environment. The user's motion becomes the computer interface device, in contrast to systems that resort to various display devices.

The ARC consists of HMPDs, a surrounding material as well as smaller ubiquitous multishaped optical materials, and tracking systems. Because the HMPD is an optical see-through HMD, users have a natural, highresolution view of the real world. In fact, the only interface between users and the real and virtual worlds is a transparent optical combiner that serves as both a win-

\section{The virtuality continuum.}

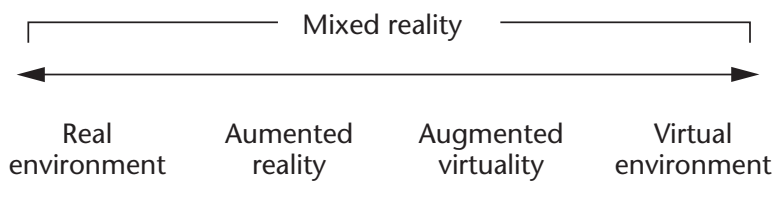

Virtual continuum 


\section{MagicBook}

Billinghurst and his colleagues first realized the idea of transitions along the virtuality continuum. ${ }^{1}$ They illustrated this concept through the MagicBook, a virtual environment that lets users become part of an interactive story. In the story, multiple users gather around a book. As they progress through the pages, they perceive 3D scenes and people via head-mounted displays.

The users can switch between two viewing displays, one allowing them to perceive the $3 \mathrm{D}$ scene as if it were resting on the open page (augmented reality), and the other allowing them to be immersed within the 3D scene (virtual reality). The head-mounted display used in the augmented reality experience was a video see-through display, where users perceived the book through miniature video cameras. The display for the immersive experience was a handheld eyepiece-type HMD, which was equipped with a switch that let users change their perspective while viewing the virtual world. Users could also remove the HMD to view the real world or replace it with another display for the mixed reality experience.

The MagicBook provides an experience where users have the capability and the incentive to travel from real to fully immersive environments within the same application. It also points convincingly to the need to develop unique technologies that span the virtual continuum.

\section{Reference}

1. M. Billinghurst, H. Kato, and I. Poupyrev, "The MagicBook: Moving Seamlessly between Reality and Virtuality," IEEE Computer Graphics and Applications, vol. 21, no. 3, May/June 2001, pp. 6-8.

dow to the real world and a medium that can combine real and virtual objects.

The HMPD is unique in the sense that it uses extremely lightweight and compact (less than 7 grams per eye) projection optics to provide computer-generated images to users with a field of view (FOV) of at least 50 degrees. Also, the HMPD doesn't compromise other important parameters, such as pupil size, which is $12 \mathrm{~mm}$ in the current HMPD design.

Some HMDs might require larger pupil sizes such as $17 \mathrm{~mm}$ to avoid vignetting the image, which might occur due to system weight and natural eye movements. Vignetting refers to the unexpected darkening of part of the perceived image. The heavier the HMD's optics, the larger the pupil size must be to avoid vignetting. In the HMPD, the combination of the extremely lightweight optics and the somewhat diffractive nature of the optical material allow for smaller pupil sizes than are typically required in other HMDs.

The retroreflective, optical material placed in the real environment lets users view computer-generated objects embedded in the environment. The material directs the stereoscopic image pairs projected from the HMPD back to the eyes of the user, thus allowing 3D perception. The material is flexible and can be used to partially or fully surround users or to cover surfaces or objects of various shapes within the surrounded environment.

\section{From technology to virtual experiences}

When users enter the ARC (see Figure 2), curved panels surround them. Users can sit at a real table covered with optical material and partially surrounded with head balls painted with optical material, as Figure 3 illustrates. Three-dimensional sound devices also provide

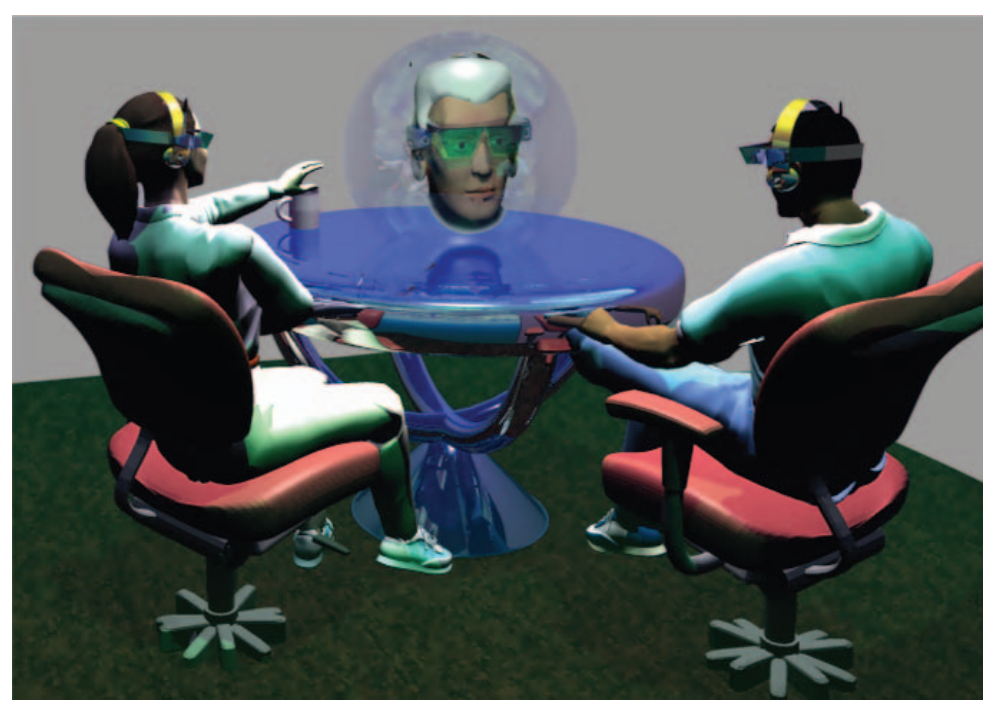

3 Users within the ARC have turned their body toward a real silvered ball painted with optical material and are engaged in an augmented reality experience with a remote user. 

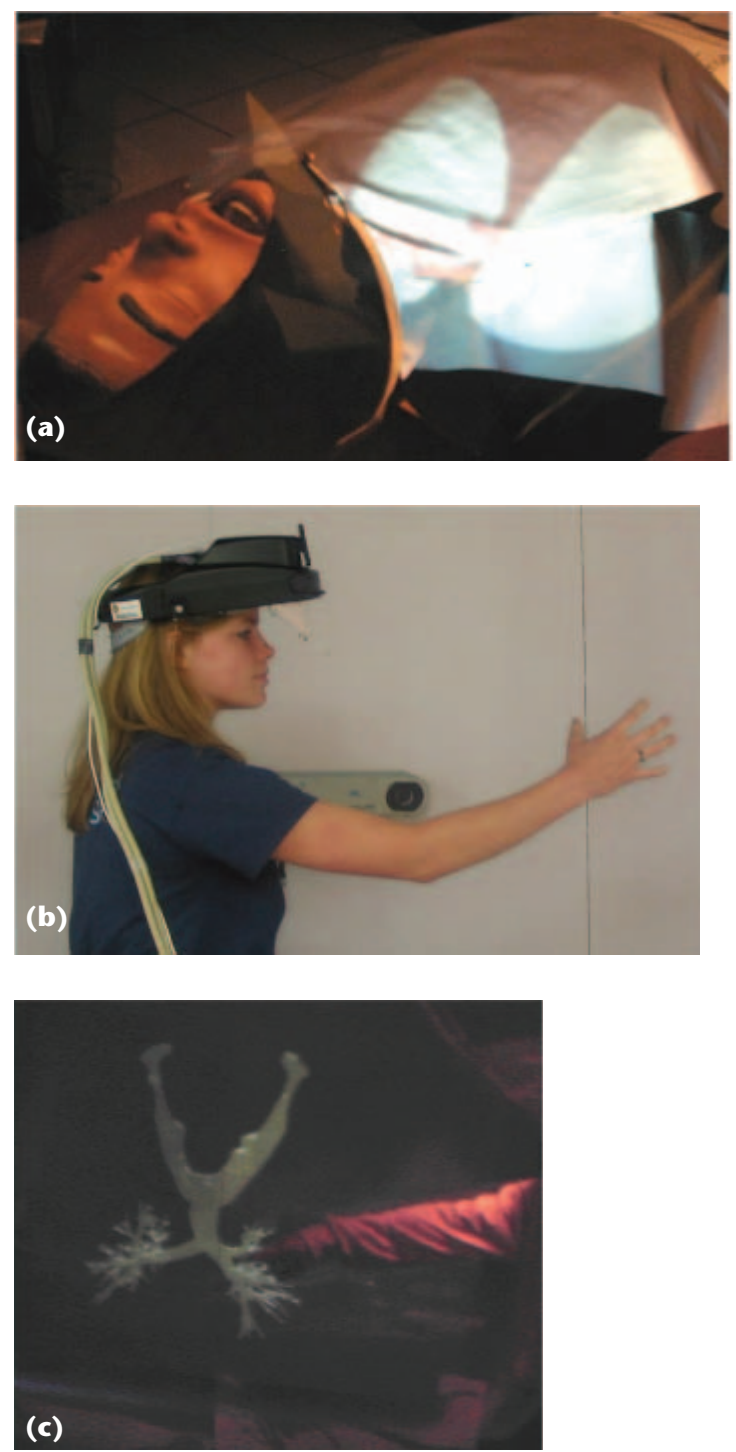

4 (a) A user experiencing augmented reality (in the ARC) on a Human Patient Simulator used in training medics on various emergency procedures. (b) A user standing in the ARC studies a floating 3D model of the combined mandible and trachea of a (c) segmented 3D visible human data set.

localized audio cues within the environment and haptic clothing such as gloves or vests might also be available to users to enable multiple experiences within the multidimensional virtuality continuum.

In Figure 3, users engage in a face-to-face real-world discussion. If users look at the table or at the silvered balls surrounding the table, they might perceive a virtual world, thus experiencing augmented reality. If users turn their bodies away from the table and look toward the surrounding screen while still perceiving some others in their FOV, they start immersing themselves in vir- tuality. Floating objects might appear to multiple users who might interact with the 3D models using multimodal technology. If a user partially occludes the view of another user by pointing at a virtual object, the occluding arm appears to be part of the virtual world, thus creating an augmented virtuality experience. If users look directly at the walls of the ARC, where all other users are outside their FOV, the users can individually experience full immersion within a virtual world. In this manner, users can span the virtuality continuum by positioning themselves in the ARC and looking at different places within the ARC. Figure 4 shows additional examples of the ARC technology.

\section{Discussion and future directions}

A technology that lets users experience multiple levels of immersion by the simple motion of their bodies within the environment is appealing and powerful. We plan to expand the ARC to enable multiple remotely networked ARCs. From an educational and training standpoint, the virtual experience needn't be limited to anatomical models. For an entertainment experience, users could explore any location of interest-be it a museum, a celebrity's house, the moon, or a fantasy world. Entertainment applications could include video games, where multiple levels of immersion could dramatically enhance turn-based strategy games and firstperson action games.

\section{Acknowledgments}

We thank Robert Banks for his assistance with the design of the ARC and Peter Hancock for funding the room structure. We thank the US Army Simulation, Training, and Instrumentation Command (STRICOM) and the NSF/EIA-99-86051 and NSF/ITR IIS-00-82016 for their invaluable support for this research.

\section{References}

1. P. Milgram and F. Kishino, "A Taxonomy of Mixed Reality Visual Displays," IECE Trans. Information and Systems (Special Issue on Networked Reality), vol. E77-D, no. 12, Dec. 1994, pp. 1321-1329.

2. H. Hua et al., "Engineering of Head-Mounted Projective Displays," Applied Optics, vol. 39, no. 22, Aug. 2000, pp. 3814-3824.

Readers may contact Jannick Rolland at the School of Optics/CREOL, University of Central Florida, 4000 Central Florida Blvd., Orlando, FL 32816-2700, email jannick@odalab.ucf.edu..

Readers may contact the department editors by email atrosenblu@ait.nrl.navy.mil or michael_macedonia@ stricom.army.mil. 